$\mathrm{e}^{+} \mathrm{e}^{-}$Collisions from Phi to Psi 2013 (PHIPSI2013)

International Journal of Modern Physics: Conference Series

Vol. 35 (2014) 1460435 (6 pages)

(C) The Authors

DOI: $10.1142 /$ S2010194514604359

\title{
STATUS AND PROSPECTS OF THE MUONIUM EXPERIMENT AT J-PARC
}

\author{
H. A. TORII $*$ K. S. TANAKA, M. TAJIMA, T. MIZUTANI, Y. MATSUDA \\ Graduate School of Arts and Sciences, University of Tokyo \\ 3-8-1 Komaba, Meguro-ku, Tokyo 153-8902, Japan
}

Y. FUKAO, H. IINUMA, Y. IKEDO, R. KADONO, N. KAWAMURA, A. KODA, K. KOJIMA, T. MIBE, Y. MIYAKE, K. NAGAMINE $\dagger$ K. NISHIYAMA, R. OHKUBO, T. OGITSU, N. SAITO, K. SASAKI, K. SHIMOMURA, P. STRASSER, M. SUGANO, A. TOYODA, K. UENO, A. YAMAMOTO, M. YOSHIDA

KEK; 1-1 Oho, Tsukuba, Ibaraki 305-0801, Japan

K. ISHIDA, M. IWASAKI, O. KAMIGAITO, S. KANDA D. TOMONO $§$ RIKEN; 2-1 Hirosawa, Wako, Saitama 351-0198, Japan

Y. UENO, K. KUBO

International Christian University (ICU); Mitaka, Tokyo 181-8585, Japan

$$
\text { M. AOKI }
$$

Osaka University; Toyonaka, Osaka 560-0043, Japan

E. TORIKAI

University of Yamanashi; Kofu, Yamanashi 400-8511, Japan

D. KAWALL

University of Massachusetts Amherst, MA 01003-9337, USA

Published 18 December 2014

A microwave spectroscopy experiment of muonium atoms are being prepared at J-PARC in Japan, aiming at an improved relative precision at a level of $10^{-8}$ in determination of

\footnotetext{
*E-mail of the corresponding author (Hiroyuki A. Torii): torii@radphys4.c.u-tokyo.ac.jp

$\dagger$ Affiliation: also at RIKEN, Japan.

${ }^{\ddagger}$ Affiliation: also at Graduate School of Science, University of Tokyo, Japan.

$\S$ Current affiliation: Kyoto University, Japan.

This is an Open Access article published by World Scientific Publishing Company. It is distributed under the terms of the Creative Commons Attribution 3.0 (CC-BY) License. Further distribution of this work is permitted, provided the original work is properly cited.
} 
the muonic magnetic moment. A major improvement of statistical uncertainty is due to higher muon intensity of the pulsed beam at J-PARC, while further improvements are expected for systematic uncertainties. Reduction of sources of systematic uncertainties are being studied: those arising from microwave power fluctuations, magnetic field inhomogeneity, muon stopping distribution and gas-density shift of resonance frequencies. Status and prospects of studies and developments of the experiment is presented in this paper.

\section{Introduction}

Muonium is a hydrogen-like exotic atom made up of a positive muon $\left(\mu^{+}\right)$and an electron. The energy level diagram is analogous to that of hydrogen, with a difference that the hyperfine splitting is 3 times as large. Purely leptonic muonium is more suited for precision spectroscopy than the hydrogen, where uncertainty of the proton radius prevents accurate comparison between spectroscopy experiments and theoretical calculations. Precision microwave spectroscopy of the ground-state hyperfine states (GS-HFS) of muonium atoms gives a rigorous test of quantum electrodynamics (QED) as well as precise determination of the magnetic moment $\left(\mu_{\mu}\right)$ of muon and hence its mass $\left(m_{\mu} \propto \mu_{\mu}{ }^{-1}\right)$. The anomalous magnetic moment $(g-2)$ is an important physical quantity for the test of the Standard Model and beyond, in the field of fundamental particle physics. The ratio of magnetic moments between muon and proton is an indispensable input parameter in deducing the $(g-2)_{\mu}$ value out of their measurements. Precision HFS spectroscopy of muonium is thus of great importance also from this point of view.

The current values of those fundamental constants of muon are determined by the latest experiment at Los Alamos, ${ }^{1}$ already 15 years ago. Our new experiment planned in coming years aims at a precision at a level of $10^{-8}$ in determination of the muonic magnetic moment, i.e. several times better than before.

\section{Overview of experimental methods}

The ground state hyperfine splitting (HFS) of $\Delta \nu=4.4 \mathrm{GHz}$ is to be measured with microwave. We do not directly measure this transition at zero magnetic field, but instead, follow the strategy of our predecessor and chose $1.7 \mathrm{~T}$ magnetic field to measure two spin-flip resonance frequencies among 4 magnetic sub-levels. Figure 1 shows the Breit-Rabi diagram of the muonium atom. Two magnetic transitions $\nu_{12}$ and $\nu_{34}$ has an important feature that the sum is constant irrespective of the magnetic field and equals the HFS at zero field i.e. $\Delta \nu=\nu_{12}+\nu_{34}$, while the difference $\nu_{12}-\nu_{34}$ is directly related to $\mu_{\mu}$.

Figure 2 shows a schematic of the experiment. Intense muon beams at J-PARC will be stopped in krypton gas to form muonium atoms $(\mathrm{Mu})$ via the electron capture process or the charge exchange reaction $\mu^{+}+\mathrm{Kr} \rightarrow \mathrm{Mu}+\mathrm{Kr}^{+}$. The muon spin is originally $100 \%$ polarized antiparallel to the direction of the muon beam, due to the left-handedness of $\nu_{\mu}$ in the decay of pions: $\pi^{+} \rightarrow \mu^{+}+\nu_{\mu}$. A microwave field will be applied in the RF cavity inside the gas chamber, flipping the muon spin. 


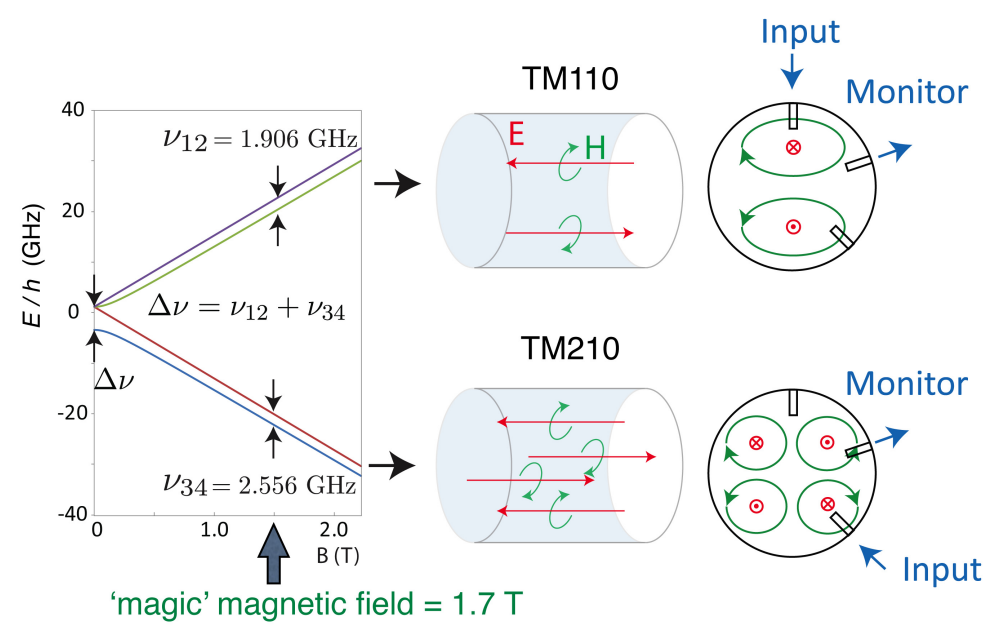

Fig. 1. Left: the Breit-Rabi diagram of the muonium atom shows the energy levels of 4 sublevels for varied magnetic field. We chose the 'magic' magnetic field of 1.7 T. Right: TM110 and TM210 modes of the cylindrical cavity were chosen so that the mode frequencies match the resonance frequencies $\nu_{12}$ and $\nu_{34}$, respectively.

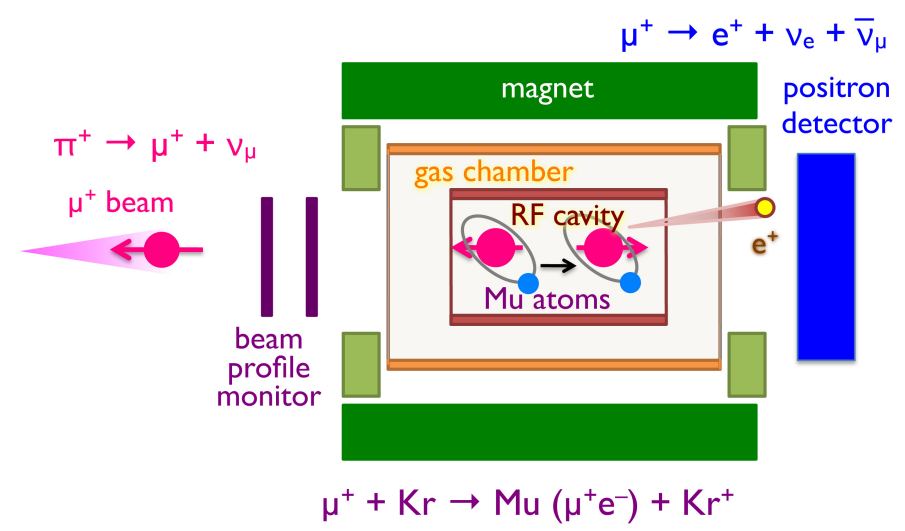

Fig. 2. A schematic of the experiment. Muon beams are stopped in Kr gas to form muonium atoms $\left(\mathrm{Mu}=\mu^{+} e^{-}\right)$in the magnetic field. Resonant muon-spin flip can be detected from asymmetry in the direction of emitted positrons.

The resonance manifests itself as an directional asymmetry of energetic positrons emitted in favor of the direction of the muon spin when the muon decays, i.e. $\mu^{+} \rightarrow e^{+}+\nu_{e}+\bar{\nu}_{\mu}$.

J-PARC MUSE $\mathrm{H}$ line is expected to provide $1 \times 10^{8}$ muons per second in pulses at $25 \mathrm{~Hz}$, and the total number would be $1 \times 10^{15}$ in a running beam time of 100 days, which means 100 times more statistics than the Los Alamos experiment. Since the statistical uncertainty was the largest component of the total experimental 


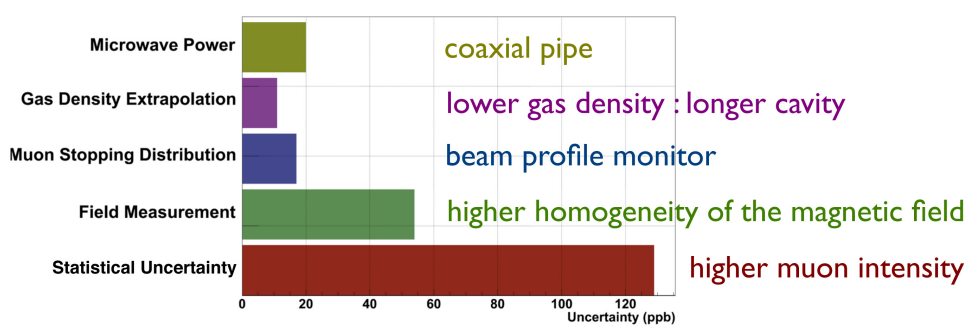

Fig. 3. The experimental uncertainty in the Los Alamos experiment was dominated by statistical uncertainty which can be improved with the high-intensity beam at J-PARC. Systematic sources of uncertainty includes magnet field inhomogeneity, muon stopping distribution and microwave power.

uncertainty there, as shown in Fig. 3, our advantage of higher statistics would surely improve the precision greatly.

\section{Details of experimental apparatuses}

We will use TM110 and TM210 microwave modes of a cylindrical cavity, and the magic magnetic field of $1.7 \mathrm{~T}$ was chosen so that the ratio between the two mode frequencies should match the ratio of resonance frequencies $\nu_{12} / \nu_{34}$. The inner diameter of the cavity is automatically determined as $187 \mathrm{~mm}$. We have made a prototype of the cavity out of copper with frequency tuning adjustability by physically moving tuning bars made of alumina. We are testing it with satisfactory results.

The axial length of the cavity can be determined arbitrarily without affecting the resonance frequencies. We chose $304 \mathrm{~mm}$ length, in comparison to $160 \mathrm{~mm}$ for the Los Alamos experiment. The longer cavity allows more reliable measurements at lower gas pressures. It is generally known that atomic collision against surrounding atoms in a gas shifts the resonance frequency, and the shift has in fact been measured for $\mathrm{Mu} \mathrm{HFS}$ in past experiments. Measurements at lower pressures are preferable, in order to minimize the uncertainty in determining the intrinsic resonance frequency by extrapolation to the zero density limit. Los Alamos measurements were performed at around 0.7 and 1.4 atm, but our long cavity will allow measurements at as low as 0.3 atm while keeping $94 \%$ of the muon stopping distribution inside the cavity.

The muon position will be monitored by a set of beam profile monitors being developed and tested. Scintillator strips of not more than 100-200 $\mu \mathrm{m}$ thickness needs to be used in order to allow the muon beam at $4 \mathrm{MeV}$ to penetrate through. For the same reason, the windows of the cavity and the gas chamber have to be made thin. Test has already been done for $100 \mu \mathrm{m} \mathrm{Al}$ foil and $25 \mu \mathrm{m} \mathrm{Cu}$ foils with $100 \mathrm{~mm}$ diameter.

The gas temperature needs to be controlled to a sub-kelvin level and the pressure to be monitored accurately. Gas purity is required to a ppm level so as to avoid 
chemical quenching of muonium polarization and possible collisional shift of the resonance frequency.

Choice of a longer cavity imposes severer requirement on the magnet. Field homogeneity of less than $1 \mathrm{ppm}$ with absolute calibration is required in a large spheroidal region of $200 \mathrm{~mm}$ diameter $\times 300 \mathrm{~mm}$ length. The field strength are measured by water NMR probes (which is the reason why the magnetic moment is measured as a ratio with respect to that of proton) at some 500 different positions to obtain a field map. The field will be corrected by shim coils and insertion of iron shims iteratively until the homogeneity is satisfactory.

The muon spin at its decay is detected by measuring the directional asymmetry of the emitted positron. In order to cope with the high event rate characteristic of the pulsed beam, highly segmented scintillators and multi-pixel photon counters (MPPCs) will be used, and the signals are processed by ASIC-based ASD and FPGA multi-hit TDC. A prototype has been developed and beam test is being performed.

\section{Estimation of uncertainty}

It is really a challenge to determine the centroid of the $100-\mathrm{kHz}$-wide resonance line to a level of a hertz. Selection of long-lived muonium (the so-called 'old muonium' method) gives narrower resonances because of the principle that longer observation time gives less uncertainty in energy, but at the sacrifice of statistics. Great improvement in the muon number at J-PARC is expected to reduce the major component of the current uncertainty limit, but several other systematic uncertainties need to be verified to aim at a precision of $10^{-8}$ for $\mu_{\mu}$.

As listed in Fig. 3, the microwave power has a direct impact on the resonance intensity. Naïvely speaking, $1 \%$ change of the power would result in $1 \%$ change of the resonance signal (unless the resonance is saturated). Any power fluctuation or drift during the course of the resonance scan would immediately deteriorate the resolution of the resonance frequency. Also, the position distribution of muonium atoms is of importance because the microwave field strength is different from position to position.

So we performed a series of simulations where the maps of muon distribution, microwave field and detection efficiency are combined. Resonant transition probability was calculated for each position to obtain a simulated resonance line for various situations. The data were fitted with theoretical lines, and the resultant centroid of the fit gave the index of uncertainty estimation. For example, when the power was gradually changed by $0.1 \%$ while the frequency was scanned over the resonance region of $500 \mathrm{kHz}$, the resultant centroid deviated from the original value by $49 \mathrm{~Hz}$. The narrower 'old muonium' spectrum turned out more robust and the shift was $5.5 \mathrm{~Hz}$. Another simulation checked the centroid deviation under the assumption that the muonium position shifted by a few centimeters during the frequency scan. All these types of realistic simulations under way help indispensable understanding of the systematic uncertainties in determination of the resonance frequencies. 
H. A. Torii et al.

\section{Summary}

In summary, we are making steady progress in preparation of muonium hyperfine spectroscopy. Development is on-going in various parts of our experiment, as described in this paper with an emphasis on the evaluation of systematic uncertainties.

\section{Reference}

1. W. Liu et al., Phys. Rev. Lett. 82 (1999) 711. 